\title{
Isolation of Vibrio alginolyticus from a patient with chronic otitis media: Report of case and review of biochemical activity
}

\author{
JOHN J. HASYN, M.S., S.M.(AAM) \\ THEODORE P. MAUER, D.O. \\ Philadelphia, Pennsylvania \\ ROBERT WARNER, D.O. \\ Allentown, Pennsylvania \\ CHRISTOPHER VON HAKE, D.O. \\ Pekin, Illinois
}

This halophilic organism was isolated from the middle ear fluid specimen from a 22-year-old man with a long-standing history of asthma and chronic otitis media with recurring infections, who related that he had recently been swimming in the Atlantic Ocean. Although this uncommon pathogen seldom is implicated in ear infections, it should be cultured for when the patient has a history of swimming in salt water. Infections caused by $V$. alginolyticus usually are self limiting, but in this case the organism was found to be resistant to the penicillins and vancomycin, antibiotics that are commonly employed in the treatment of otitis media. The patient responded well to chloramphenicol therapy. The biochemical activity of the isolate and the most useful microbiologic characteristics for differentiating $V$. alginolyticus from the other Vibrio species are discussed in depth.

The etiologic agent of cholera, Vibrio cholerae Ogroup-1, historically has been the vibrio organism of greatest interest to physicians and microbiologists. Initially, other vibrios were not considered to be clinically significant. They were categorized as nonagglutinable vibrios (NAG), because they did not agglutinate in V. cholerae O-group-1 antiserum, or as noncholera vibrios (NCV).

It was not until the NCV/NAG group was subdivided into separate species, which occurred in the mid-1930s, that it became apparent that several of these, including $V$. parahemolyticus, $V$. vulnificus, the non-O-group 1 portion of $V$. cholera species, and $V$. alginolyticus, are often pathogenic for humans. ${ }^{1}$
In addition, two Vibrio species, group F (EFG) Vibrios and V. mestchnikovii, are of uncertain pathogenicity for humans. Six of 9 halophilic Vibrio species have been named since $1979 .^{2}$ The clinical features, ecologic niches, pathogenic mechanisms, and epidemiologic characteristics of vibrios other than O-group $1 \mathrm{~V}$. cholerae are described elsewhere. . $^{3-5}$

The purpose of this case study is to report an unusual isolate, $V$. alginolyticus, from a patient with chronic otitis media. Microbiologists usually encounter one of the following microorganisms from properly collected ear specimens from patients with otitis media: Streptococcus pneumoniae, Hemophilus influenzae, Streptococcus pyogenes, Staphylococcus aureus, or Branhamella catarrhalis. ${ }^{6}$ Halophilic vibrios (those that require a high concentration of salt for optimal growth) such as $V$. alginolyticus have only recently been recognized as human pathogens, usually after the patient's exposure to seawater.

\section{Report of case}

A 22-year-old white man had a long-standing history of asthma triad (asthma, nasal polyps, and sensitivity to aspirin), as well as chronic serous otitis media with recurrent infection. The patient was admitted in November 1984 through the Short Procedure Unit. Preadmission testing showed all parameters to be within normal limits. Symptoms prior to hospitalization included fullness in ear, drainage, and conductive hearing loss.

The patient previously had recurrent serous otitis media with associated asthma and nasal polyps, with secondary chronic sinusitis. The pressure equalization tube was clogged and nonfunctioning prior to surgery. Surgery was indicated to remove apparent granulation tissue and replace pressure equalization tube, which would allow further medical treatment to be successful.

The patient was taken to the operating room, and bilateral myringotomy, insertion of pressure equalization tubes, right middle ear exploration, removal of granulation tissue, and removal of a Goode tube from the right ear were performed. At surgery, the middle ear mucosa 


\begin{tabular}{ll|}
\hline \multicolumn{2}{l}{ TABLE 1 . BIOCHEMICAL CHARACTERISTICS OF VIBRIO AL- } \\
GINOLYTICUS ISOLATE. & \\
\hline Test & Reaction \\
\hline Growth on thiosulfate citrate bile & Yellow colonies \\
salts (TCBS) a agar & \\
Growth in chocolate agar & Positive \\
Growth on MacConkey a agar & Positive \\
Growth on sheep blood agar & Positive (swarming) \\
Hemolysis on sheep blood agar & Negative \\
Cytochrome oxidase & Positive \\
Catalase & Positive \\
Anaerobic dextrose & Positive \\
Aerobic dextrose & Positive \\
Maltose & Positive \\
Lactose & Negative \\
Sucrose & Positive \\
Xylose & Negative \\
Salicin & Negative \\
Arginine dihydrolase & Negative \\
Lysine decarboxylase & Positive \\
Ornithine decarboxylase & Positive \\
Urease & Negative \\
ONPG & Negative \\
Indole & Negative \\
Nitrate & Positive \\
Phenylalanine deaminase & Negative \\
Citrate & Negative \\
Motility & Positive \\
Acetate & Negative \\
Voges-Proskauer & Positive* \\
Triple sugar iron agar & †cid/acid \\
Deoxyribonuclease & Positive \\
Sodium chloride (NaCL) tolerance: & \\
Growth in 0\% NaCL & Negative \\
Growth in 1\% NaCL & Positive \\
Growth in 3\% NaCL & Positive \\
Growth in 6\% NaCL & Positive \\
Growth in 8\% NaCL & Positive \\
Growth in 10\% NaCL & Positive (weak \\
& response) \\
*Response was positive when 3\% NaCL & was added to the culture \\
medium. \\
tAcid/acid indicates the fermentativeaction of the organism on the 3 \\
sugars present in the test medium: glucose, lactose, and/or sucrose. \\
\hline
\end{tabular}

was more edematous than previously noted and granulation tissue was present in the area of the incision site. The surgery was well tolerated by the patient, and he was discharged later in the day. He was given Cortisporin Otic Suspension and instructed to use 4 drops in each ear 4 times a day.

A right middle ear fluid specimen had been submitted to the microbiology laboratory for aerobic culture. After 18 hours' incubation, a pure culture of $V$. alginolyticus was observed on 5-percent sheep blood agar, chocolate agar, and MacConkey agar plates. After 48 hours, both $V$. alginolyticus and Staphylococcus coagulase negative were isolated from broth culture. Further questioning of the patient revealed that he had recently been swimming in the Atlantic Ocean along the New Jersey coast. He was treated successfully with chloramphenicol. Chloramphenicol was selected because of its broad-spectrum coverage for $V$. alginolyticus and Staphylococcus coagulase negative as determined on in vitro antimicrobial susceptibility testing. The role of the Staphylococcus co-
TABLE 2. BAUER-KIRBY DISK DIFFUSION SUSCEPTIBILITIES OF VIBRIO ALGINOLYTICUS ISOLATE.

\begin{tabular}{|ll|}
\hline Antibiotic & Susceptibility \\
\hline Methicillin & Resistant \\
Ampicillin & Resistant \\
Carbenicillin & Resistant \\
Penicillin & Resistant \\
Piperacillin & Resistant \\
Cephalothin & Susceptible \\
Cefamandole & Susceptible \\
Cefoxitin & Susceptible \\
Cefotaxime & Susceptible \\
Cefoperazone & Susceptible \\
Amikacin & Susceptible \\
Gentamicin & Susceptible \\
Chloramphenicol & Susceptible \\
Erythromycin & Susceptible \\
Trimethoprim-sulfamethoxazole & Susceptible \\
Vancomycin & Resistant \\
Tobramycin & Susceptible \\
\hline
\end{tabular}

agulase negative in the infection was questionable, because it was isolated in broth culture only after 48 hours of incubation.

The $V$. alginolyticus isolate was initially identified with the Minitek Nonfermenter System (BBL, Cockeysville, Maryland). Like other halophilic vibrios, the isolate fermented glucose, was positive for oxidase and lysine decarboxylase, and was motile (Table 1) Distinguishing the isolate from other halophilic vibrios were the facts that it fermented sucrose and not lactose, and that it grew in a 10-percent sodium chloride solution. Gram's staining revealed curved, gram-negative rods. In vitro susceptibility testing determined the isolate to be resistant only to the penicillins and vancomycin (Table 2). The isolate was submitted to a reference laboratory for confirmation of identification.

The most useful microbiologic characteristics for differentiating $V$. alginolyticus from the other Vibrio species are fermentation of sucrose, negative fermentation reaction for lactose, growth in 10-percent sodium chloride solution, negative O-nitrophenyl-B-D-galactopyranoside, swarming on 5-percent sheep blood agar plate, and positive Voges-Proskauer reaction. It should be noted that the Voges-Proskauer reaction was initially negative when the Minitek Nonfermenter identification system was used; however, the reaction was positive when the Voges-Proskauer medium was supplemented with 3percent sodium chloride.

\section{Discussion}

There have been only a few reported instances of isolation of $V$. alginolyticus from the ear, ${ }^{3,5,8}$ but it has been implicated as an etiologic agent of otitis externa and otitis media. The organism primarily has been isolated from feces and skin. ${ }^{8}$ Its role in infectious diarrhea has not been demonstrated; the organism has been isolated repeatedly from asymptomatic individuals who had ingested raw seafood. However, V. alginolyticus has been in- 
creasingly implicated in skin infections, including abscesses, cellulitis, and ulcers.

$V$. alginolyticus usually has been isolated in mixed cultures. Exposure to marine environments usually antedates infection, although in at least one report ${ }^{7}$ this setting could not be confirmed. Unlike $V$. vulnificus and some of the other pathogenic halophilic vibrios, infections caused by $V$. alginolyticus typically are self limiting, with response to no treatment or only to local therapy in the normal host. In striking contrast to the highly invasive $V$. vulnificus, bacteremia has rarely been reported with $V$. alginolyticus. ${ }^{9}$

\section{Summary}

Vibrio species other than V. cholera have been associated with disease. The organisms are increasingly being recognized as human pathogens in parts of the world where cholera is not endemic. $V$. alginolyticus is one such microorganism that should be considered as an etiologic agent of otitis media and wound infections from patients using coastal water facilities for either occupational or recreational purposes. Thorough history taking is therefore essential if the organism is to be considered an infectious agent.

1. Wilson, G.S., and Miles, A. eds. Topley and Wilson's Principles of bacteriology, virology, and immunity. Ed. 6. Williams and Wilkins, Baltimore, 1975

2. Morris, J.G., Jr., and Black, R.B.: Cholera and other Vibrioses in the United States. N Engl J Med 312:343-50, 7 Feb 85

3. Blake, P.A., Weaver, R.E., and Hollis, D.G.: Diseases of humans (other than cholera) caused by vibrios. Annu Rev Microbiol 34:341-67, 1980

4. McTighe, A.H.: Pathogenic vibrio species. Isolation and identification. Lab Management 20:43-6, Aug 82

5. WHO Scientific Working Group: Cholera and other Vibrio associated diarrhea. Bull WHO 58:353-74, 1980

6. Nelson, J.D.: Otic infections. In A modern treatise of infectious processes, edited by P.D. Hoeprich, et al. Harper and Row, Philadelphia, 1983, pp. $1385-90$

7. Hansen, W., Crokaert, F., and Yourassowsky, E.: Two strains of Vibrio species with unusual biochemical features isolated from ear tracts. $\mathrm{J}$ Clin Microbiol 9:152-3, Jan 79

8. Schmidt, U., Chmel, H., and Cobbs, C.: Vibrio alginolyticus infections in humans. J Clin Microbiol 10:666-8, Nov 79

9. Karnaugh, L.: Vibrio vulnificus. Neptune's revenge? Diagn Med :21-5, Jun 84

Mr. Hasyn is microbiology laboratory supervisor, Department of Pathology and Laboratory Medicine, Philadelphia College of Osteopathic Medicine, Philadelphia. Dr. Mauer is chairman of the Department of Otorhinolaryngology at PCOM. Dr. Warner was a resident in that department when the paper was written. $\mathrm{He}$ is now an attending physician, Allentown Osteopathic Hospital, Department of Otorhinolaryngology, Allentown, Pennsylvania. Dr. Von Hake was chief resident in the Department of Otorhinolaryngology at PCOM when the paper was written. He is now a member of the attending staff, Pekin Memorial Hospital, Department of Otolaryngology.

Mr. Hasyn, Philadelphia College of Osteopathic Medicine, Department of Pathology and Laboratory Medicine. 4150 City Avenue, Philadelphia, Pennsylvania 19131. 